\title{
Cost analysis of inpatient treatment of anorexia nervosa in adolescents: hospital and caregiver perspectives
}

\author{
Alene Toulany MD, Matthew Wong RN, Debra K. Katzman MD, Nadia Akseer MSc, \\ Cathleen Steinegger MD MSc, Rebecca L. Hancock-Howard PhD, Peter C. Coyte PhD
}

Abstract

Background: Admission to hospital is the treatment of choice for anorexia nervosa in adolescent patients who are medically unstable; however, stays are often prolonged and frequently disrupt normal adolescent development, family functioning, school and work productivity. We sought to determine the costs of inpatient treatment in this population from a hospital and caregiver perspective, and to identify determinants of such costs.

Methods: We used micro-costing methods for this cohort study involving all adolescent patients (age 12-18 yr) admitted for treatment of anorexia nervosa at a tertiary care child and adolescent eating disorder program in Toronto, between Sept. 1, 2011, and Mar. 31, 2013. We used hospital administrative data and Canadian census data to calculate hospital and caregiver costs.

Results: We included 73 adolescents in our cohort for cost-analysis. We determined a mean total hospital cost in 2013 Canadian dollars of \$51 349 (standard deviation [SD] \$26 598) and a mean total societal cost of \$54 932 (SD \$27 864) per admission, based on a mean length of stay of 37.9 days (SD $19.7 \mathrm{~d}$ ). We found patient body mass index (BMI) to be the only significant negative predictor of hospital cost $(p<0.001)$. For every unit increase in BMI, we saw a $15.7 \%$ decrease in hospital cost. In addition, we found higher BMI $(p<0.001)$ and younger age $(p<0.05)$ to be significant negative predictors of caregiver costs.

Interpretation: The economic burden of inpatient treatment for adolescents with anorexia nervosa on hospitals and caregivers is substantial, especially among younger patients and those with lower BMI. Recognizing the symptoms of eating disorders early may preclude the need for admission to hospital altogether or result in admissions at higher BMls, thereby potentially reducing these costs.

\section{A}

norexia nervosa is the third most common chronic condition affecting adolescent girls, with an incidence of about $5 \% .^{1-3}$ The effects of anorexia nervosa on the physical and psychological health of adolescents are profound. ${ }^{2,45}$ Anorexia nervosa has the highest mortality ${ }^{6}$ of the psychiatric disorders and causes serious medical complications. ${ }^{7,8}$ Recommendations for the management of anorexia nervosa suggest inpatient admission to hospital because of medical instability, failure of outpatient treatment or suicidal ideation. ${ }^{2,9-11}$

Hospital admissions for adolescents with anorexia nervosa are often prolonged and can disrupt normal adolescent development, school, social life and family functioning. Caregivers and families of patients with eating disorders present with high levels of psychological distress and burden..$^{12}$ In addition, parents describe the illness as financially draining, making reference to the hidden costs of chronic illness and stays in hospital such as those associated with transportation, food, additional family care and time missed from work..$^{13}$
The economic impact of eating disorders is substantial but notably under-researched. ${ }^{14}$ Comprehensive evaluations of direct and indirect costs are lacking. ${ }^{14,15}$ Although admissions to hospital for adolescents with eating disorders have a substantial effect on the public health system, few studies have examined the costs associated with pediatric inpatient treatment. ${ }^{16-20}$ We aimed to describe the hospital and caregiver costs for inpatient treatment of adolescent anorexia nervosa from a Canadian perspective and assess the predictors of admission cost in this pediatric population.

Competing interests: None declared.

This article has been peer reviewed.

Correspondence to: Alene Toulany, alene.toulany @ sickkids.ca

CMAJ Open 2015. DOI:10.9778/cmajo.20140086 


\section{Methods}

\section{Study setting and population}

Using our eating disorder program's administrative database, we identified all adolescent patients (age 12-18 yr) with anorexia nervosa admitted to a large, tertiary care academic pediatric hospital in Toronto, between Sept. 1, 2011, and Mar. 31, 2013. We chose this period because of substantial changes in leadership and clinical practice in the program before Sept. 1, 2011, and because administrative cost data were not available after Mar. 31, 2013. The diagnosis of anorexia nervosa was based on criteria from the Diagnostic and Statistical Manual of Mental Disorders, 4th edition (DSM-IV), psychometric tests and comprehensive clinical assessments done by a team of eating-disorder experts comprising adolescent medicine specialists, psychologists and dietitians. We excluded patients if they had incomplete costing data. Patients with more than 1 admission to hospital during the study period had only their first stay included in the analysis.

We collected data on patient age, sex, postal code of residence, presence and age of siblings, body mass index (BMI), comorbidities, duration of anorexia nervosa symptoms, previous treatments for anorexia nervosa, length of stay in hospital and time spent on the wait list from hospital charts.

The study was approved by the research ethics board at The Hospital for Sick Children, Toronto.

\section{Study design}

We used micro-costing data collection methods ${ }^{21}$ to estimate the cost of anorexia nervosa-related hospital admissions from hospital and caregiver perspectives. We calculated admission costs from patient-level data and extracted from hospital administrative data for each patient's total length of stay in hospital (Appendix 1, available at www.cmajopen.ca/content/3/2/E192 /suppl/DC1).

\section{Hospital costs}

Physicians at the study site are salaried employees and do not bill the provincial Ministry of Health directly for reimbursement for patient care; however, the physicians conduct "shadow billing" for administrative purposes. We obtained these shadow billings from hospital administrative data for review by 2 of the investigators (A.T. and M.W.). Reviewers classified missing data or illogical costs (i.e., shadow billings that were too low for a given length of stay) as inaccurate and replaced these data with estimates determined using the Ontario Physician Schedule of Benefits (2013).22 For the cost categories of personnel labour, medications, laboratory investigations, diagnostic imaging and food, our primary sources were patient-level administrative and financial data obtained through the hospital's decision-support division. Overhead hospital costs considered in the cost analysis included housekeeping and utilities, which were based on the square-footage cleaned or occupied, and laundry services, which were based on kilograms of linens cleaned. ${ }^{23,24}$

\section{Caregiver costs}

Caregiver costs, representing time costs incurred as a result of an admission to hospital for anorexia nervosa treatment, were estimated by determining lost work productivity and lost leisure time of caregivers, rather than that of the adolescent patient, because patients were still in school and not embedded in the workforce. No standard method for calculating the economic cost of time missed from school exists, so we did not include this cost. To calculate the amount of caregiver time lost from work and leisure, expert opinion was sought from experienced eating-disorder care providers at the study site (D.K., C.S. and A.T.). We assumed that, each week, caregivers would attend 1 health care team meeting and 7 meal supports or visits.

We estimated caregiver costs associated with hospital visits by entering each patient's postal code into Google Maps at about 1700 (the time parents usually visit to assist with meals) to determine the round-trip distance (in kilometers) and time spent in traffic (in hours). We then used the Ontario commuter cost of $\$ 0.16 / \mathrm{km}$ in 2011 multiplied by the number of kilometers travelled to obtain a measure of travel costs (including fuel and maintenance costs) per visit. ${ }^{25}$ Parking costs were based on hospital parking rates of $\$ 6$ per half-hour (\$20 maximum) or \$3 per half-hour (\$7 maximum) for either a weekday or weekend visit. We based caregiver costs associated with team meetings on the assumption that it is common for caregivers to spend 1 hour in the meeting and an additional 30 minutes visiting their child. Because family meetings occur during weekday working hours, we assumed that caregivers required time off from work to attend. A conservative measure of 1.5 hours and time spent in traffic was used to calculate the time lost from work. We estimated the caregiver cost for lost work productivity per team meeting using the product of this time measure and mean household incomes for each family's postal code. ${ }^{26}$

The quantification of caregiver costs incurred from hospital visits were considered lost leisure time. We used the human capital method to calculate the cost of lost leisure time using the current market wage in Ontario (minimum wage $\$ 10.25 / \mathrm{h}$ ), as previously discussed by Tranmer and colleagues. ${ }^{27,28} \mathrm{We}$ assumed that family visits were 2 hours, plus time spent in traffic. If patients had siblings less than 12 years of age, an additional babysitter cost was included for these visits (quantified at the minimum wage for students aged $<18 \mathrm{yr}$ in Ontario: $\$ 9.60 / \mathrm{h}) .^{28}$

\section{Societal costs}

We calculated the societal costs for inpatient treatment of adolescents with anorexia nervosa by determining the sum of hospital and caregiver costs.

\section{Statistical analysis}

We described patient characteristics across the entire sample. We summarized discrete and categorical variables using frequencies and proportions; we examined continuous variables using means, medians, standard deviations and ranges. We used Pearson correlation coefficients and Student $t$ tests, as appropriate, to evaluate initial bivariate associations between cost outcomes and predictors. Because hospital and caregiver cost outcomes were highly right skewed, we used log-transformation for their statistical analyses. We used multivariate linear regression models to determine the adjusted impact of relevant predictors 


\section{OPEN}

Research

on the dependent cost variables. We used the best subsets model-building strategy to determine the most parsimonious and powerful model, with the highest adjusted $R^{2}$. We examined multicollinearity among predictors using variance inflation factors; we considered variables with variance inflation factors greater than or equal to 4 to be multicollinear variables. ${ }^{29} \mathrm{We}$ consulted studentized residuals, leverage points and Cook distance values to examine potential outliers and influential observations. We assessed the linearity of continuous variables using residual versus predictor plots. We used SAS version 9.3 (SAS Institute Inc.) for all statistical analyses, with statistical significance held at $p$ less than 0.05 .

\section{Results}

Of the 95 adolescents who were admitted to hospital because of anorexia nervosa during the study period, 73 met the inclusion criteria. The other 22 patients were excluded owing to incomplete administrative costing data. The mean age of participants was 15.2 (standard deviation [SD] 1.6) years (Table 1). Most of the participants were female (89\%). The mean BMI at admission was 16.4 (SD 2.5), with a median of 16.1 (range 11.3-26.2). Most patients $(n=64,88 \%)$ were either admitted to the in- patient unit directly from the emergency department or transferred to the unit from another hospital. Only $12 \%$ of participants $(n=9)$ spent time on the wait list after referral. Before admission to hospital, the mean duration of symptoms was 55.8 (SD 59.9) weeks, with a median of 41.6 (range 4.4-362.9) weeks. Thirty percent of participants had at least 1 comorbid medical diagnosis, and 34\% had at least 1 comorbid psychiatric diagnosis at admission.

The mean length of hospital stay was 37.9 (SD 19.7) days, with a median of 35 (range 9-153) days. Table 2 shows the mean and median values for total hospital and caregiver costs in 2013 Canadian dollars (US\$1.00 = Can\$1.03 in 2013).$^{30}$ Based on the mean length of stay of 37.9 days, we estimated the hospital, caregiver and total societal costs to be about $\$ 1355, \$ 95$ and $\$ 1450$ per admission day, respectively.

\section{Hospital costs}

Multivariate linear regression analyses showed that the model with the highest adjusted $R^{2}$ was able to predict $18.3 \%$ of variance in hospital costs per admission $(p<0.001)$ and included BMI and wait time between referral and inpatient treatment as predictors (Table 3). Body mass index at admission was the only individually significant predictor of hospital costs $(p<0.001)$. For

Table 1: Characteristics of study cohort $(n=73)$

\begin{tabular}{|lc|}
\hline Characteristic & No. (\%) of patients* \\
\hline Age, yr, mean \pm SD (median, range) & $15.2 \pm 1.6(15.4,12.0-17.6)$ \\
\hline Female sex & $65(89)$ \\
\hline Comorbid medical diagnosis at admission & $22(30)$ \\
\hline Comorbid psychiatric diagnosis at admission & $25(34)$ \\
\hline Admitted directly from emergency department & $64(88)$ \\
\hline BMl at admission, mean \pm SD (median, range) & $16.4 \pm 2.5(16.1,11.3-26.2)$ \\
\hline Duration of symptoms, wk, mean \pm SD (median, range) & $55.8 \pm 59.9(41.6,4.4-362.9)$ \\
\hline Length of stay, d, mean \pm SD (median, range) & $37.9 \pm 19.7(35,9-153)$ \\
\hline Household income by postal code, CAD, mean \pm SD (median, range) & $83752 \pm 33415(82795,30979-207589)$ \\
\hline Distance travelled between home and hospital, km, mean \pm SD (median, range) & $39.6 \pm 33.4(31.8,2.7-134.0)$ \\
\hline $\begin{array}{l}\text { Note: BMI = body mass index, CAD = Canadian dollars, SD = standard deviation. } \\
\text { *Unless otherwise indicated. }\end{array}$ & \\
\hline
\end{tabular}

Table 2: Estimates of total hospital and caregiver costs

\begin{tabular}{|c|c|c|c|}
\hline \multirow[b]{2}{*}{ Variable } & \multicolumn{3}{|c|}{ Total cost, ${ }^{*} \$$} \\
\hline & Mean \pm SD & & dian (range) \\
\hline Hospital cost & $51349 \pm 26598$ & 47304 & 5 790-194 618) \\
\hline Caregiver cost & $3583 \pm 1904$ & 3146 & $(673-11$ 119) \\
\hline Lost work productivity & $766 \pm 389$ & 673 & (93-2 080) \\
\hline Lost leisure time & $2817 \pm 1588$ & 2565 & (531-9 039) \\
\hline Societal cost $†$ & $54932 \pm 27864$ & 49807 & 6 488-201 036) \\
\hline
\end{tabular}


every unit increase in BMI, we observed a $15.7 \%$ decrease in the mean hospital cost for admission (about \$3350), after controlling for wait time between referral and start of inpatient treatment. We also tested a second model that included previous anorexia nervosa treatments and comorbid medical conditions. Body mass index remained the only significant predictor, even after we controlled for these additional covariates, and the overall additional variance explained by this model was minimal and not significant. Therefore, we retained the original 2-predictor model.

We identified 3 outliers in the final model. We detected no differences in our results when outliers were included or excluded; therefore, we included outliers in the final model.

\section{Caregiver costs}

Multivariate linear regression revealed an original model with best-adjusted $R^{2}$ explaining $26.2 \%$ of variance in caregiver costs per admission. In this model, BMI at admission was the only significant predictor after we controlled for wait time between referral and start of inpatient treatment, age and other comorbid psychiatric conditions. We identified 2 outliers; when they were excluded from the model, age at admission became a significant predictor. We decided to exclude these outliers from the final analysis to ensure model validity.

The final model included 3 predictors that explained $31.8 \%$ of the total variance for caregiver costs per admission $(p<0.001)$, with both age at admission $(p<0.05)$ and BMI at admission $(p<0.001)$ found to be statistically significant after we controlled for wait time between referral and start of inpatient treatment (Table 4). For every unit increase in age, we observed a $13.6 \%$ decrease in mean caregiver cost after controlling for BMI at admission and wait time; this equated to a decrease of about $\$ 125$. For every unit increase in BMI, we observed a $23 \%$ decrease in caregiver cost after controlling for age at admission and wait time (about \$370). Sensitivity analyses to test the robustness of our findings were performed using lower and higher estimates for overall caregiver costs, lost work productivity, cost of visiting and childcare costs. These analyses did not change our findings.

\section{Interpretation}

The results of our study suggest that, from a societal perspective, the economic burden of admissions to hospital for the treatment of anorexia nervosa is substantial, with a mean cost of \$54 932 per admission and a mean length of stay of 37.9 days. Our multivariate linear regression model predicting hospital costs showed a strongly significant negative association with $\mathrm{BMI}$ at admission $(p<0.001)$. When predicting caregiver costs, however, the multivariate linear regression model showed significant negative associations with both $\mathrm{BMI}(p<0.001)$ and age at admission $(p<0.05)$.

Inpatient care costs are the most expensive component of care for patients with eating disorders. ${ }^{20,31}$ Cost comparisons between our findings and results from the literature are difficult to perform owing to the different perspectives used in cost estimation, varying cost calculation methods, heterogeneous study populations and vastly different health care systems and sources of finance. ${ }^{16,18-20,31,32}$

Table 3: Multivariate linear regression model for hospital costs per admission to hospital for anorexia nervosa in adolescent patients

\begin{tabular}{|c|c|c|c|c|}
\hline Predictor variable & $\begin{array}{l}\text { Logarithmic cost } \\
\text { model (SE) }\end{array}$ & $\begin{array}{c}\text { Calculated marginal } \\
\text { change, } \%\end{array}$ & $\begin{array}{l}\text { Approximate marginal } \\
\text { cost change, } C A D^{*}\end{array}$ & $p$ value \\
\hline Intercept & $11.979 \quad(0.317)$ & NA & NA & $<0.001$ \\
\hline Wait time between referral and treatment & $-0.0170(0.0103)$ & -3.8 & -902.45 & 0.10 \\
\hline BMI at admission & $-0.0740(0.0191)$ & -15.7 & -3346.82 & $<0.001$ \\
\hline
\end{tabular}

Table 4: Multivariate linear regression model for caregiver costs per admission to hospital for anorexia nervosa in adolescent patients

\begin{tabular}{|c|c|c|c|c|}
\hline Predictor variable & $\begin{array}{l}\text { Logarithmic cost } \\
\text { model (SE) }\end{array}$ & $\begin{array}{c}\text { Calculated marginal } \\
\text { change, } \%\end{array}$ & $\begin{array}{l}\text { Approximate marginal } \\
\text { cost change, } C A D^{\star}\end{array}$ & $p$ value \\
\hline Intercept & $10.909 \quad(0.585)$ & NA & NA & \\
\hline Wait time between referral and treatment & $-0.0194(0.0105)$ & -4.8 & -67.01 & 0.07 \\
\hline Age at admission & $-0.0632(0.0314)$ & -13.6 & -123.08 & $<0.05$ \\
\hline BMI at admission & $-0.113 \quad(0.0222)$ & -23.0 & -364.38 & $<0.001$ \\
\hline
\end{tabular}


Using a national database of health insurance claims from the United States, Striegel-Moore and colleagues determined that the inpatient treatment cost for female adolescents with anorexia nervosa was US\$17384 for a mean length of stay of 26 days. ${ }^{31}$ Krauth and colleagues conducted a cost-of-illness study for eating disorders among patients 15-24 years old in Germany from the perspective of the health insurance schemes; they found the mean cost of a hospital stay to be $€ 12800$ for a mean length of stay of 50 days. ${ }^{18}$ In contrast, substantially higher costs were reported in the United States by Kalisvaart and Hergenroeder (US\$97 231 for a mean length of stay of $50 \mathrm{~d}$ ). ${ }^{19}$ Our estimated mean cost of about $\$ 1355$ per day falls between these previously published cost estimates for inpatient adolescents with anorexia nervosa.

The finding that a higher BMI at admission was predictive of lower hospital and caregiver costs is not surprising. The primary goal of admission to hospital for adolescents with anorexia nervosa is nutritional rehabilitation and weight gain. Patients who present with a higher BMI have shorter hospital stays to attain a healthy weight. ${ }^{33,34}$ Although length of stay can be viewed only as a proxy for the costs of admission to hospital, both outcomes are strongly correlated and describe resource consumption by the patient. ${ }^{32,35}$

Older age at admission has been correlated with longer hospital stays in young adults with anorexia nervosa. ${ }^{33}$ In our study, younger age at admission resulted in increased caregiver costs. This may be because caregivers spent more time in hospital, and subsequently less time at work, supporting their younger adolescent. In addition, parents of younger adolescents with anorexia nervosa may be more likely to have other young children who require childcare in their absence, resulting in increased caregiver costs. Finally, younger adolescents with anorexia nervosa may have less of a buffer for malnutrition and, therefore, require more intensive nutritional rehabilitation and longer stays in hospital to restore weight and meet the physical demands of a developing body.

\section{Strengths and limitations}

This micro-costing analysis provides a comprehensive estimate of direct and indirect costs for admissions to hospital for the treatment of anorexia nervosa in adolescent patients. Our access to patient-level costing data allowed us to use a "bottom-up approach" as opposed to the less precise and more commonly described "top-down approach." We are limited, however, in our ability to accurately capture caregiver costs owing to the retrospective nature of the study. Data on caregivers' incomes, time spent in hospital, lost work productivity and transportation costs were based on median neighbourhood income quintiles in addition to assumptions from experience and observations of eating-disorder specialists providing front-line care at the study site. Having a child admitted to hospital likely affects caregivers' work productivity in ways that were unaccounted for in the study, and we have probably underestimated this economic burden. In addition, we relied heavily on hospital administrative data and the accuracy of the hospital's data collection, cost calculation and accounting practices.

\section{Conclusion}

Body mass index and age were significant predictors of hospital and caregiver costs for inpatient treatment of anorexia nervosa in adolescent patients, and these costs were substantial. Recognizing the symptoms of eating disorders early may preclude the need for admission to hospital altogether or result in admissions at higher BMIs, thereby potentially reducing these costs. Admitting patients at higher BMIs could also translate into better patient outcomes, ${ }^{36}$ because low weight at referral has been previously associated with poor prognosis. ${ }^{19,37}$ Whether earlier admission is cost-effective in the long term when compared with outpatient family-based treatment, however, has yet to be established and should be the focus of future research.

\section{References}

1. Fisher M, Golden NH, Katzman DK, et al. Eating disorders in adolescents: a background paper. 7 Adolesc Health 1995;16:420-37.

2. Golden NH, Katzman DK, Kreipe RE, et al. Eating disorders in adolescents: position paper of the Society for Adolescent Medicine. 7 Adolesc Health 2003; 33:496-503.

3. Lucas AR, Beard CM, O'Fallon WM, et al. 50-year trends in the incidence of anorexia nervosa in Rochester, Minn.: a population-based study. Am 7 Psychiatry 1991;148:917-22.

4. Treasure J, Claudino AM, Zucker N. Eating disorders. Lancet 2010; 375:583-93.

5. Becker AE, Grinspoon SK, Klibanski A, et al. Eating disorders. $N$ Engl 7 Med 1999;340:1092-8.

6. Arcelus J, Mitchell AJ, Wales J, et al. Mortality rates in patients with anorexia nervosa and other eating disorders. A meta-analysis of 36 studies. Arch Gen Psychiatry 2011;68:724-31.

7. Katzman DK. Medical complications in adolescents with anorexia nervosa: a review of the literature. Int 7 Eat Disord 2005;37:Suppl:S52-9; discussion S87-9.

8. DiVasta AD, Walls CE, Feldman HA, et al. Malnutrition and hemodynamic status in adolescents hospitalized for anorexia nervosa. Arch Pediatr Adolesc Med 2010;164:706-13.

9. Rosen DS. Identification and management of eating disorders in children and adolescents. Pediatrics 2010;126:1240-53.

10. Beumont P, Hay P, Beumont D, et al. Australian and New Zealand clinical practice guidelines for the treatment of anorexia nervosa. Aust NZ J Psychiatry 2004;38:659-70.

11. Watson HJ, Bulik CM. Update on the treatment of anorexia nervosa: review of clinical trials, practice guidelines and emerging interventions. Psychol Med 2013; 43:2477-500.

12. Zabala MJ, Macdonald P, Treasure J. Appraisal of caregiving burden, expressed emotion and psychological distress in families of people with eating disorders: a systematic review. Eur Eat Disord Rev 2009;17:338-49.

13. Hillege S, Beale B, McMaster R. Impact of eating disorders on family life: individual parents' stories. 7 Clin Nurs 2006;15:1016-22.

14. Simon J, Schmidt U, Pilling S. The health service use and cost of eating disorders. Psychol Med 2005;35:1543-51.

15. Stuhldreher N, Konnopka A, Wild B, et al. Cost-of-illness studies and costeffectiveness analyses in eating disorders: a systematic review. Int 7 Eat Disord 2012;45:476-91.

16. Robergeau K, Joseph J, Silber TJ. Hospitalization of children and adolescents for eating disorders in the state of New York. 7 Adolesc Health 2006;39:806-10.

17. Byford S, Barrett B, Roberts C, et al. Economic evaluation of a randomised controlled trial for anorexia nervosa in adolescents. Br $\mathcal{F}$ Psychiatry 2007;191: 436-40.

18. Krauth C, Buser K, Vogel H. How high are the costs of eating disorders anorexia nervosa and bulimia nervosa - for German society? Eur 7 Health Econ 2002;3:244-50.

19. Kalisvaart JL, Hergenroeder AC. Hospitalization of patients with eating disorders on adolescent medical units is threatened by current reimbursement systems. Int 7 Adolesc Med Health 2007;19:155-65.

20. Lock J, Couturier J, Agras WS. Costs of remission and recovery using family therapy for adolescent anorexia nervosa: a descriptive report. Eat Disord 2008; 16:322-30.

21. Frick KD. Microcosting quantity data collection methods. Med Care 2009;47(Suppl 1):S76-81.

22. Schedule of benefits for physician services under the Health Insurance Act. 2013. Toronto: Ontario Ministry of Health and Long-Term Care; 2013. Available: www.health.gov.on.ca/english/providers/program/ohip/sob/physserv/ physserv_mn.html (accessed 2014 Dec. 1)

23. Canadian patient cost database technical document: MIS patient costing methodology, November 2011. Ottawa: Canadian Institute for Health Information; 2011. 
Available: www.cihi.ca/CIHI-ext-portal/pdf/internet/MIS_PATIENT_COST_ METH_EN (accessed 2014 Dec. 1).

24. Drummond MFSM, Torrance GW, O'Brien BJ, et al. Cost analysis. In: Methods for the economic evaluation of health care programmes. 3rd ed. New York: Oxford University Press; 2005:55-102.

25. Reimbursement for business use of personal vebicles model year 2011 update. Ottawa: National Joint Council of the Public Service of Canada; 2011. Available: www.njc-cnm.gc.ca/aux bin.php? auxid=581 (accessed 2014 Dec. 1).

26. 2011 National Household Survey. Ottawa: Statistics Canada; 2011. Available: www12.statcan.gc.ca/nhs-enm/index-eng.cfm?HPA (accessed 2014 Dec. 1).

27. Tranmer JE, Guerriere DN, Ungar WJ, et al. Valuing patient and caregiver time: a review of the literature. Pharmacoeconomics 2005;23:449-59.

28. Minimum wage. Toronto: Ontario Ministry of Labour; 2013. Available: www .labour.gov.on.ca/english/es/pubs/guide/minwage.php (accessed 2014 Dec. 1).

29. O'Brien R. A caution regarding rules of thumb for variance inflation factors. Qual Quant 2007;41:673-90.

30. Financial markets department year average of exchange rates. Ottawa: Bank of Canada; 2013. Available: www.icao.int/CAFICS/News\%20Library/nraa-2013. pdf (accessed 2014 Dec. 1).

31. Striegel-Moore RH, Leslie D, Petrill SA, et al. One-year use and cost of inpatient and outpatient services among female and male patients with an eating disorder: evidence from a national database of health insurance claims. Int 7 Eat Disord 2000;27:381-9.

32. Haas L, Stargardt T, Schreyoegg J, et al. Inpatient costs and predictors of costs in the psychosomatic treatment of anorexia nervosa. Int 7 Eat Disord 2012; 45:214-21.

33. Nozoe S, Soejima Y, Yoshioka M, et al. Clinical features of patients with anorexia nervosa: assessment of factors influencing the duration of in-patient treatment. 7 Psychosom Res 1995;39:271-81.

34. Maguire S, Surgenor LJ, Abraham S, et al. An international collaborative database: its use in predicting length of stay for inpatient treatment of anorexia nervosa. Aust NZ7 Psychiatry 2003;37:741-7.

35. Schwartz WB, Mendelson DN. Hospital cost containment in the 1980s. Hard lessons learned and prospects for the 1990s. NEngl 7 Med 1991;324:1037-42.

36. Fichter MM, Quadflieg N, Hedlund S. Twelve-year course and outcome predictors of anorexia nervosa. Int 7 Eat Disord 2006;39:87-100.

37. Hebebrand J, Himmelmann GW, Wewetzer C, et al. Body weight in acute anorexia nervosa and at follow-up assessed with percentiles for the body mass index: implications of a low body weight at referral. Int 7 Eat Disord 1996; 19:347-57.

Affiliations: Division of Adolescent Medicine, Department of Paediatrics (Toulany, Katzman, Steinegger), The Hospital for Sick Children; Institute of Health Policy, Management and Evaluation (Toulany, HancockHoward, Coyte), University of Toronto; Lawrence S. Bloomberg Faculty of Nursing (Wong), University of Toronto, Toronto, Ont.; University of Toronto (Toulany, Katzman, Steinegger); Child Health Evaluative Sciences, Research Institute (Katzman, Akseer), The Hospital for Sick Children; Dalla Lana School of Public Health (Akseer), University of Toronto, Toronto, Ont.

Contributors: All of the authors contributed to the study design and the analysis and interpretation of the data. Alene Toulany and Matthew Wong performed the data collection and drafted the initial manuscript. All of the authors were involved with critical revisions of the manuscript, approved the final version to be published and agreed to act as guarantors of the work.

Acknowledgements: The authors thank Ladan Dadgar, Ethel Lagman, Shelley Todd and Nina Corcoran for their help in providing hospital administrative and costing data.

Supplemental information: For reviewer comments and the original submission of this manuscript, please see www.cmajopen.ca/content/3/2/E192 /suppl/DC1 